\title{
Stochastic Bandwidth Estimation in Networks with Random Service
}

\author{
Ralf Lübben, Markus Fidler, Jörg Liebeherr
}

\begin{abstract}
Numerous methods for available bandwidth estimation have been developed for wireline networks and their effectiveness is well-documented. However, most methods fail to predict bandwidth availability reliably in a wireless setting. It is accepted that the increased variability of wireless channel conditions makes bandwidth estimation more difficult, however, a (satisfactory) explanation why these methods are failing is missing. This paper seeks to provide insights into the problem of bandwidth estimation in wireless networks, or, more broadly, in networks with random service. We express bandwidth availability in terms of bounding functions with a defined violation probability. Exploiting properties of a stochastic min-plus linear system theory, the task of bandwidth estimation is formulated as inferring an unknown bounding function from measurements of probing traffic. We present derivations showing that simply using the expected value of the available bandwidth in networks with random service leads to a systematic overestimation of the traffic departures. Furthermore, we show that in a multi-hop setting with random service at each node, available bandwidth estimates requires observations over (in principle infinitely) long time periods. We propose a new estimation method for random service which is based on iterative constant rate probes that take advantage of statistical methods. We show how our estimation method can be realized to achieve both good accuracy and confidence levels. We evaluate our method for wired single- and multi-hop networks, as well as for wireless networks.
\end{abstract}

\section{INTRODUCTION}

The objective of available bandwidth estimation is to infer the service offered by a network path from traffic measurements taken at end systems only. In bandwidth estimation methods, end systems exchange timestamped probe packets, and study the dispersion of these packets after they have traversed a network of nodes. In recent years, available bandwidth estimation has attracted significant interest and a wide variety of measurement tools and techniques have been developed, e.g., [16]-[18], [34], [39], [41]. Many of the most popular methods for available bandwidth estimation are based on congestion-inducing packet trains, where a packet train consists of a sequence of probe packets. By sending packet trains at a rate exceeding the available bandwidth, the network becomes congested, thereby imprinting information on the network state on the dispersion of probe packets.

Virtually all available bandwidth methods were developed for wireline networks, where communication channels consist

R. Lübben and M. Fidler are with the Department of Electrical Engineering and Computer Science, Leibniz Universität Hannover. J. Liebeherr is with the Department of Electrical and Computer Engineering, University of Toronto.

The work of R. Lübben and M. Fidler is supported by a Research Grant and in part by an Emmy Noether Grant from the German Research Foundation (DFG), respectively. The work of J. Liebeherr is supported in part by an NSERC Strategic Project. This manuscript is an extended version of a paper that appears in the IEEE Infocom 2011 proceedings. of fixed-capacity links, and where the available bandwidth of a link is given by its unconsumed capacity. Some of these methods have been adapted for wireless networks (see Sec. II), in particular WiFi networks, however, they generally lack the robustness and reliability achieved in fixed-capacity wireline environments. A potential source of errors are unsuitable model assumptions. For example, many methods interpret the packet dispersion under the assumption that probe traffic flows through one or more fixed-capacity FIFO links that experience cross traffic [16], [34], [41]. Even though FIFO queueing may be highly prevalent in wired network infrastructures today, FIFO assumptions are difficult to justify in wireless multiaccess networks [5], [6], [38]. Another widely used model assumption is that networks are work-conserving, i.e., they generate output whenever traffic is ready for transmission. However, as pointed out in [23], latencies incurred during channel access lead to non-work-conserving systems.

In this paper we investigate fundamental difficulties of measuring the available bandwidth in wireless networks with congestion-inducing packet trains. Rather than revising or adapting wireline approaches to wireless channels, e.g., by trying to eliminate superimposed random 'noise', we seek to develop from the ground up a new modeling and inference approach for networks that are subject to randomness of both traffic and transmission channels. We dispense with the modeling assumption of a work-conserving queueing system, and, taking advantage of concepts from the stochastic network calculus [20], replace it with that of a general stationary system.

The point of departure of our efforts is a recent systemtheoretic approach of bandwidth estimation [28]. Here, the network is viewed as a time-invariant deterministic system where throughput and delays of traffic are governed by an unknown bounding function, referred to as service curve. Service curves can express work-conserving as well as non-work-conserving systems. For example, the function $\mathcal{S}(t)=R \max \{0, t-T\}$ expresses a latency-rate service curve of a non-work-conserving system with a latency of $T$ and rate $R$. It can be argued that existing congestion inducing probing methods assume that a network is a time-invariant deterministic system. As a case in point, packet train probing schemes similar to Pathload [17] and Pathchirp [39] were shown to be aligned with a system-theoretic interpretation in that they can reliably extract the shape of convex service curves of a time-invariant deterministic system. A strength of the system-theoretic approach is that service curves offer a natural extension to networks with several bottleneck links, by exploiting properties of the network calculus [8], [26]. 
A limitation of this system-theoretic approach is the assumption that the measured system is time-invariant deterministic, meaning that identical packet probes, e.g., sent as packet pairs or packet trains, sent at two different time instances experience the same backlog or delay. Even in networks with random traffic load or random link capacities, this can be an appropriate model as long as the time scale of measurements is small compared to the time scale at which resource availability in the network changes. In such cases, the network can be viewed as a state-dependent deterministic system, where a single sample of the available bandwidth can be interpreted as being conditioned on the current state of the network. Evaluating a large number of samples corresponds to computing a conditional average of the system state. However, when network or traffic characteristics change on a short time scale, a time-invariant and deterministic system characterization is not suitable.

The main contribution of this paper is the development of a system-theoretic approach to bandwidth estimation of systems with random service, which can account for variability due to statistical properties of network traffic and transmission channels on short time scales. We develop the foundations for the approach (in Sec. III) by first expressing random service in a network by $\varepsilon$-effective service curves from the stochastic network calculus [7]. Working within the framework of the network calculus, we show that it is feasible to compute an $\varepsilon$-effective service curve of a network from packet trains with fixed inter packet gap in each train [16], [17], [34]. By relating the service curves of the network calculus to a common definition of available bandwidth, we can express limitations of existing probing methods in a stochastic stationary system. For example, for a single-link network the service curve describing the unused capacity on a link coincides with the commonly used definition of available bandwidth. However, in a system with random service, the expected value of the available bandwidth provides only an optimistic estimate of the traffic departures. For multi-link networks we show that the definition of end-to-end available bandwidth can be recovered only as a time limit, and that the available bandwidth may overestimate the usable service over short time scales.

Equipped with a system theory for bandwidth estimation of stochastic systems, we address the development of a practical probing scheme (in Sec. IV). For example, a challenge of using packet train probing in a stochastic system is that packet trains may push the system into a non-stationary state. In principle, observing the system in a stationary state requires packet trains with infinite length. We show that, in practice, it is possible to detect stationarity with finite packet trains using stationarity tests and other statistical methods, and dynamically adapt packet trains to the required length. We also study the required lengths of packet trains and the required number of repeated measurements.

Using measurement results from a controlled testbed, we quantify the effect of variability on the estimated service and observe the impact of the burstiness of cross traffic, access delays, and retransmissions on service availability. We present measurement results for wired single-hop and multihop networks as well as for wireless networks.
An implication of our study is that the widely used assumption of work-conserving fixed-capacity FIFO links with cross traffic can be replaced by a more general network model without specific requirements on the multiplexing method. This may open the field of bandwidth estimation to network environments where FIFO or work-conserving assumptions are not justified.

The remainder of this paper is structured as follows. In Sec. II, we discuss related work on approaches to bandwidth estimation, with a focus on congestion-inducing methods. In Sec. III, we derive a stochastic min-plus approach for estimating networks with random service. In Sec. IV we consider practical aspects of bandwidth estimation of a stochastic system. In Sec. V, we provide an experimental validation of our method. Sec. VI gives brief conclusions.

\section{Available BAndwidth Estimation}

The term available bandwidth denotes the capacity that is left unused by other traffic in the network, referred to as cross traffic. For a link $h$ it can be expressed for any time interval $(\tau, t]$ as [29]

$$
\alpha^{h}(\tau, t)=\frac{1}{t-\tau} \int_{\tau}^{t} C^{h}\left(1-u^{h}(x)\right) d x,
$$

where $C^{h}$ is the (possibly time-varying) capacity of the link and $u^{h}(t) \in[0,1]$ is its utilization by cross traffic at time $t$. For cross traffic with a long-term average rate $\lambda^{h}$, the limit $\liminf _{\delta \rightarrow \infty} \alpha^{h}(\tau, \tau+\delta)=C^{h}-\lambda^{h}$ is referred to as long-term available bandwidth. The end-to-end available bandwidth of a network path is frequently defined as the minimum of the available bandwidths of all traversed links $(h=1, \ldots, H)$ [29], [30]

$$
\alpha^{n e t}(\tau, t)=\min _{h}\left\{\alpha^{h}(\tau, t)\right\} .
$$

\section{A. Bandwidth Estimation of Work-Conserving Systems}

Many bandwidth estimation tools assume a fluid timeinvariant network model with work-conserving FIFO scheduling, where the relation between the incoming rate $r_{I}$ and the outgoing rate $r_{O}$ of a constant bit rate (CBR) probe at a link, referred to as rate response curve [29], [35], is given by

$$
\frac{r_{I}}{r_{O}}= \begin{cases}1 & , r_{I} \leq C-\lambda \\ \frac{r_{I}+\lambda}{C} & , r_{I}>C-\lambda .\end{cases}
$$

Random cross traffic is often interpreted as distorting the traffic dispersion given by response curves. To eliminate the random distortions, estimation tools have applied averaging [16], [41], linear regression [34], and Kalman filtering [11]. For packet pair probes, the corresponding function describing the dispersion of the probes, is called gap response curve [16], [29]. Several works have extended the deterministic CBR traffic model for the response curve at a FIFO system to stochastic ones, e.g., [10], [14], [29], [30], [36], [37]. A queueing theoretic framework for bandwidth estimation is analyzed in [29], where it is shown that the assumption of fluid CBR traffic generates an upper bound for the available bandwidth, and that the deviation can be resolved using packet trains of 
infinite length. The work is extended to multi-hop networks in [30]. In [10], a distribution for the output gap is derived for a general arrival process in conjunction with parameter estimation for known cross traffic distributions. Fundamental limitations of active probing are analyzed in [33] based on a queueing model of a FIFO system.

Methods which do not explicitly assume FIFO scheduling, but are compatible with this assumption are, e.g., Pathchirp [39] and Pathload [17], [18]. Both methods increase their probing rate until an increase of one-way delays, or, equivalently, queueing delays of probe packets is detected. Pathload specifies the available bandwidth as a range, to capture its time-varying nature. The underlying deterministic network model is relaxed to filter out short-term fluctuations in the detection of long-term trends.

\section{B. Bandwidth Estimation in Wireless Networks}

Bandwidth estimation in wireless networks is more difficult for a variety of reasons. Link capacities in wireless networks are not constant due to interference and time-varying channel conditions. Also, wireless networks may not behave like FIFO systems. For example, a measurement study of IEEE 802.11 networks [6] showed that the multiplexing of the Distributed Coordination Function (DCF) is similar to a fair queueing algorithm. Further, inherent delays due to media access, retransmissions, and other factors, create a non-work-conserving system [23], where the transmission channel can be idle even if traffic is pending. Proposed approaches in the literature have addressed these issues by adapting or revising bandwidth estimation methods developed for wireline networks.

Time varying link capacities are addressed in [27]. Instead of estimating the link capacity by the smallest gap between two successive probe packets, the median of a sequence of probe gaps is used. In [22] the authors observe dependencies of the cross traffic rate and the probe packet size on the capacity of IEEE 802.11 networks. A passive measurement approach to estimate the available bandwidth in IEEE 802.11 networks is presented in [24]. Non-FIFO scheduling is investigated in [5], [38] where the rate response curve is adapted to the channel access in IEEE 802.11 networks and to the wireless channel. Additionally, the transient behavior of access delays and its impact on the response curve are described in [38]. Kalman filters are adapted to the channel access and to the wireless channel in IEEE 802.11 networks in [6] and [21], respectively. Recently, attention has been given to measuring the service in wireless home networks. In addition to the rate of the available bandwidth, estimation approaches for home networks in [9] address access delays as important metrics. In [23], correlations of access delay measurements are used to differentiate, among others, traffic congestion and hidden terminal problems. The study emphasizes the need for nonwork-conserving models, due to the significant role of nonqueueing related delay components.

\section{Bandwidth Estimation of Min-Plus Linear Systems}

System theory offers an alternative model for bandwidth estimation methods [1], [15], [28]. A network is represented as a general system with traffic arrivals $A(t)$ as input, and traffic departures $D(t)$ as output of the system. $A(t)$ and $D(t)$ are non-negative and non-decreasing functions that denote the cumulative number of bits seen in an interval $(0, t]$. By convention $A(0)=0$ and, due to causality, $D(t) \leq A(t)$ for all $t \geq 0$. We sometimes use the shorthand notation $A(\tau, t)=A(t)-A(\tau)$ to denote the arrivals in the interval $(\tau, t]$. We use $B(t)=A(t)-D(t)$ to describe the backlog of the system at time $t$, and the smallest number $d$ such that $D(t+d) \geq A(t)$ to characterize the delay at time $t$.

A system is called time-invariant if a time shifted arrival function $A(t-\tau)$ results in a time-shifted departure function $D(t-\tau)$. Consider two arrival functions $A_{1}, A_{2}$, and their corresponding departure functions $D_{1}, D_{2}$ of a system. A system is called min-plus linear, when arrivals of the form $\min \left\{A_{1}(t), A_{2}(t)\right\}+c$, where $c \geq 0$ is constant, result in departures $\min \left\{D_{1}(t), D_{2}(t)\right\}+c$. The form of the arrivals can be viewed as a linear combination in an algebra, where the minimum replaces the usual addition, and the addition takes the role of the multiplication. This gives rise to the min-plus algebra formulation of the network calculus.

The behavior of any time-invariant min-plus linear system can be characterized by a non-negative and non-decreasing function $\mathcal{S}(t)$, referred to as service curve. The service curve relates departures and arrivals of a system by

$$
D(t)=\inf _{\tau \in[0, t]}\{A(\tau)+\mathcal{S}(t-\tau)\}=: A \otimes \mathcal{S}(t),
$$

where the operator $\otimes$ is the convolution under the min-plus algebra. In the language of system theory, the service curve is the impulse response of a min-plus linear system [26]. Service curves can be used to describe a wide range of systems. A work-conserving constant rate link is expressed by $\mathcal{S}(t)=R t$ $(R>0)$, and a non-work-conserving pure delay server which imposes a latency $d>0$ is given by $\mathcal{S}(t)=\infty$ if $t \geq d$ and $\mathcal{S}(t)=0$ if $t<d$.

For min-plus linear systems, bandwidth estimation can be expressed as the inversion problem of obtaining $\mathcal{S}$ from $D=A \otimes \mathcal{S}$ [28], where $A$ and $D$ are arrival and departure functions of probing traffic. A solution to the inversion problem can be obtained from constant-rate packet train arrivals $A(t)=r t$ and measurements of the departure traffic $D(t)$. Using the maximum system backlog, expressed as $B_{\max }(r)=$ $\sup _{\tau}\{A(\tau)-D(\tau)\}$, the service curve can be computed as

$$
\mathcal{S}(t)=\sup _{r \geq 0}\left\{r t-B_{\max }(r)\right\} .
$$

An advantage of a system theoretic view is a straightforward extension to multi-hop settings. Given a network of $H$ systems in sequence where $\mathcal{S}^{h}(h=1, \ldots, H)$ denotes the service curve of the $h$-th system, a service curve $\mathcal{S}^{\text {net }}$ for the entire sequence of $H$ systems is given by the min-plus convolution $\mathcal{S}^{\text {net }}=\mathcal{S}^{1} \otimes \ldots \otimes \mathcal{S}^{H}$. The equivalent replacement of a sequence of single-hop systems by a single system with service curve $\mathcal{S}^{\text {net }}$ provides a handle on an analysis of multi-hop networks.

While Eq. (4) is suitable for expressing the service offered by constant rate links, traffic regulators, or fair schedulers, it assumes linearity under the min-plus algebra since the 
convolution is a linear operation. For non-linear systems, Eq. (4) can be relaxed to provide a linear lower bound of the form $D \geq A \otimes \mathcal{S}$ [26]. Notable non-linear systems that are used in many actual networks are FIFO schedulers [13], where the service obtained by a flow in an overloaded system depends on its arrival rate, see Eq. (3). In [28], it is argued that networks can be viewed as linear systems that transition to a non-linear regime when the network becomes saturated, and it is shown that the transition can be observed using suitable non-linearity tests.

The main limitation of the system-theoretic approach to bandwidth estimation is that the measured system must satisfy time-invariance, i.e., the service curve in Eq. (4) is a deterministic function that does not depend on the absolute values of $\tau$ and $t$ but only on the length of the time interval $\delta=t-\tau$. Dispensing with this assumption requires a system-theoretic framework where the network service is a random process.

\section{INFERENCE OF A RANDOM SERVICE}

In this section, we develop the foundation for a stochastic bandwidth estimation methodology for networks with stationary random service. Starting from a system characterization by random service processes as defined in [8], we link the service processes to the stochastic network calculus [20] and to the available bandwidth as given in Eq. (1). In detail, we proceed as follows: In Sec. III-A, we show that stochastic bounds of random service processes can be expressed in terms of $\varepsilon$ effective service curves $S^{\varepsilon}$ [7] from the stochastic network calculus, which enables us to express statistical performance bounds of the type $\mathrm{P}[B>x] \leq \varepsilon$. We show how to infer an $\varepsilon$-effective service curve from measurements of probing traffic in Sec. III-B. In Sec. III-C, we address the relation of effective service curves to the available bandwidth given by Eqs. (1) and (2), and explore conditions where the available bandwidth systematically overestimates the actual departures of a network. In Sec. III-D, we show that by expressing arrivals and departures using a max-plus algebra, the inference of service can be done directly from packet timestamps. This last step will be used in the next section, where we develop a practical probing methodology.

\section{A. Systems with Random Service in the Network Calculus}

Given a system with random service, the assumption of time-invariance, on which the definition of the service curve $\mathcal{S}(t)$ in Eq. (4) is based, does not hold. To this end, we substitute $\mathcal{S}(t)$ by a bivariate random process $S(\tau, t)$, which expresses a random service experienced in the time interval $(\tau, t]$. We refer to $S(\tau, t)$ as service process. Similar as in Eq. (4), the departures of a min-plus linear random system can be related to its arrivals by the service process as [8]

$$
D(t)=\inf _{\tau \in[0, t]}\{A(\tau)+S(\tau, t)\}=: A \otimes S(t) .
$$

In the stochastic network calculus, random service can be expressed by $\varepsilon$-effective service curves, which express a non-random time-invariant bound on the service that can be violated with probability $\varepsilon$. An $\varepsilon$-effective service curve $\mathcal{S}^{\varepsilon}(t)$ specifies a service guarantee of the form [7]

$$
\mathrm{P}\left[D(t) \geq \inf _{\tau \in[0, t]}\left\{A(\tau)+\mathcal{S}^{\varepsilon}(t-\tau)\right\}\right]>1-\varepsilon .
$$

The following lemma links the definitions in Eqs. (5) and (6), in that it specifies $\varepsilon$-effective service curves as a stationary bound of a random service process.

Lemma 1: Given a system with service process $S(\tau, t)$ as in Eq. (5). Any function $\mathcal{S}^{\varepsilon}(t)$ that satisfies the sample path bound

$$
\mathrm{P}\left[S(\tau, t) \geq \mathcal{S}^{\varepsilon}(t-\tau), \forall \tau\right]>1-\varepsilon
$$

for $t \geq 0$ is an $\varepsilon$-effective service curve in the sense of Eq. (6) of the system.

The advantage of the $\varepsilon$-effective service curve $\mathcal{S}^{\varepsilon}(t-\tau)$ compared to the service process $S(\tau, t)$ is that it is timeinvariant, i.e., $\mathcal{S}^{\varepsilon}(t-\tau)$ depends on the duration $t-\tau$, but it does not depend on the location of the time interval $(\tau, t]$. Later, we will relate the service process Eq. (5) to the available bandwidth definition in Eq. (1).

Proof: Consider a sample path $S^{\omega}(\tau, t)$ of $S(\tau, t)$ and fix $t \geq 0$. If $S^{\omega}(\tau, t) \geq \mathcal{S}^{\varepsilon}(t-\tau)$ for all $\tau \in[0, t]$, it follows from the monotonicity of the min-plus convolution that

$$
D(t)=A \otimes S^{\omega}(t) \geq A \otimes \mathcal{S}^{\varepsilon}(t) .
$$

Since, by assumption, the condition $S^{\omega}(\tau, t) \geq \mathcal{S}^{\varepsilon}(t-\tau)$ holds for all $\tau \in[0, t]$ with probability at least $1-\varepsilon$, the claim is proven.

\section{B. Estimation of Effective Service Curves}

We next show how an $\varepsilon$-effective service curve can be obtained from constant rate packet train probes of the form $A(t)=r t$. To this end, we phrase the backlog as a function of the probing rate, that is, $B(r, t)=r t-D(t)$. The steady state backlog for $t \rightarrow \infty$ is abbreviated by $B(r)$. We define the quantile of the backlog distribution $B^{\xi}(r, t)$ as

$$
B^{\xi}(r, t)=\inf \{x \geq 0: \mathrm{P}[B(r, t) \leq x]>1-\xi\},
$$

where $\xi$ denotes a violation probability. With this definition, Th. 1 provides the foundation for a packet train based estimation method.

Theorem 1: Given a system with service process $S(\tau, t)$ as in Eq. (5). Select a finite set $R$ of rates $r \geq 0$. For all $t \geq 0$, the function

$$
\mathcal{S}^{\varepsilon}(t)=\max _{r \in R}\left\{r t-B^{\xi}(r)\right\}
$$

is an $\varepsilon$-effective service curve in the sense of Eq. (6) of the system with violation probability $\varepsilon=\sum_{r \in R} \xi$.

Proof: From $B(t)=A(t)-D(t)$ and Eq. (5) it follows that

$$
B(t)=\sup _{\tau \in[0, t]}\{A(\tau, t)-S(\tau, t)\} .
$$

The supremum in Eq. (8) implies that $B(t) \geq A(\tau, t)-S(\tau, t)$ for all $\tau \in[0, t]$, permitting us to write

$$
S(\tau, t) \geq A(\tau, t)-B(t), \forall \tau \in[0, t] .
$$


Inserting $A(\tau, t)=r(t-\tau)$ and using the backlog quantile yields

$$
\mathrm{P}\left[S(\tau, t) \geq r(t-\tau)-B^{\xi}(r, t), \forall \tau\right]>1-\xi .
$$

Using the complement and applying the union bound for a set of rates $R$, it follows that

$$
\mathrm{P}\left[\bigcup_{r \in R}\left\{S(\tau, t)<r(t-\tau)-B^{\xi}(r, t), \forall \tau\right\}\right] \leq \sum_{r \in R} \xi .
$$

and, hence,

$$
\mathrm{P}\left[S(\tau, t) \geq \max _{r \in R}\left\{r(t-\tau)-B^{\xi}(r, t)\right\}, \forall \tau\right]>1-\sum_{r \in R} \xi .
$$

With Lem. 1 we obtain that $\mathcal{S}^{\varepsilon}(t-\tau)$ defined as

$$
\mathcal{S}^{\varepsilon}(t-\tau)=\max _{r \in R}\left\{r(t-\tau)-B^{\xi}(r, t)\right\}
$$

for all $\tau \in[0, t]$ is an $\varepsilon$-effective service curve with violation probability $\varepsilon=\sum_{r \in R} \xi$. Letting $t \rightarrow \infty$ and inserting the steady state backlog quantile $B^{\xi}(r)$ completes the proof.

Th. 1 gives rise to a method for service curve estimation for networks with random service using packet train probes sent at different rates $r$. Each packet train provides a sample of the steady state backlog $B(r)$. Taking many samples provides an empirical distribution of the steady state backlog from which the quantile, denoted by $\widetilde{B}^{\xi}(r)$ and given in Eq. (7), is obtained. Applying Th. 1, we can compute an estimate of an $\varepsilon$ effective service curve as $\widetilde{\mathcal{S}}^{\varepsilon}(t)=\max _{r \in R}\left\{r t-\widetilde{B}^{\xi}(r)\right\}$. The choice of the probing set $R$ presents a trade-off. The accuracy of the estimate increases by adding probing rates. At the same time, adding probing rates increases the violation probability due to the use of the union bound in Th. 1 .

Since our method uses steady state backlogs $B(r)$ to obtain service curve estimates, the convergence to a steady state is vital. The following lemma is important as it provides existence of the steady state backlog distribution as long as the probing rate does not exceed the limiting rate of the service process, defined as $\liminf _{t \rightarrow \infty} \frac{S(\tau, t)}{(t-\tau)}$.

Lemma 2: Given arrivals $A(\tau, t)$ at a system with service process $S(\tau, t)$, satisfying Eq. (5), where $A(\tau, t)$ and $S(\tau, t)$ are jointly stationary in the strong sense ${ }^{1}$.

1) The backlog $B(t)$ is stochastically increasing in $t$.

2) If for all $t$ it holds that

$$
\limsup _{\delta \rightarrow \infty} \frac{A(t-\delta, t)}{\delta}<\liminf _{\delta \rightarrow \infty} \frac{S(t-\delta, t)}{\delta}
$$

almost surely, the backlog converges in distribution to a finite random variable $B$.

Note that we extended the processes $A(\tau, t)$ and $S(\tau, t)$ from $0 \leq \tau \leq t<\infty$ to $-\infty<\tau \leq t<\infty$. The lemma generalizes Lem. 9.1.4 in [8] from a constant rate server to a server with random service. The proof closely follows [8].

\footnotetext{
${ }^{1}$ Stationarity in the strong sense means that $\mathrm{P}[A(\tau, t)<x]=\mathrm{P}[A(\tau+$ $\vartheta, t+\vartheta) \leq x]$ and joint stationarity $\mathrm{P}[A(\tau, t) \leq x, S(\tau, t) \leq y]=\mathrm{P}[A(\tau+$ $\vartheta, t+\vartheta) \leq x, S(\tau+\vartheta, t+\vartheta) \leq y]$ for all $\tau \leq t$ and all $\vartheta$.
}

Proof: From Eq. (8) it follows for any $x$ and $\vartheta>0$ that

$$
\begin{aligned}
& \mathrm{P}[B(t+\vartheta) \geq x] \\
& =\mathrm{P}\left[\sup _{\tau \in[0, t+\vartheta]}\{A(\tau, t+\vartheta)-S(\tau, t+\vartheta)\} \geq x\right] \\
& \geq \mathrm{P}\left[\sup _{\tau \in[0, t]}\{A(\tau+\vartheta, t+\vartheta)-S(\tau+\vartheta, t+\vartheta)\} \geq x\right] .
\end{aligned}
$$

From the assumption of joint stationarity $A(\tau+\vartheta, t+\vartheta)-$ $S(\tau+\vartheta, t+\vartheta)$ is equal in distribution to $A(\tau, t)-S(\tau, t)$ for all $\tau, t$, and $\vartheta>0$. The last line equals $\mathrm{P}[B(t) \geq x]$ so that we get $\mathrm{P}[B(t+\vartheta) \geq x] \geq \mathrm{P}[B(t) \geq x]$, thus, proving the first claim.

For the second claim, if the given inequality holds, then there exists a finite random variable

$$
T=\sup \{\delta \geq 0: A(t-\delta, t) \geq S(t-\delta, t)\},
$$

for any $t$. Consequently, $A(t-\delta, t)<S(t-\delta, t)$ holds for all $\delta>T$ with probability one. Moreover, since $A(t-\delta, t)$ is nondecreasing in $\delta \geq 0$ we have $A(t-\delta, t)<S(t-T-\vartheta, t)$ for all $0 \leq \delta \leq T$ and any $\vartheta>0$. Combining the two statements and using that $S(t-\delta, t)$ for $\delta \geq 0$ and $S(t-T-\vartheta, t)$ are non-negative yields

$$
A(t-\delta, t)-S(t-\delta, t) \leq S(t-T-\vartheta, t)
$$

for all $\delta \geq 0$. Hence,

$$
\sup _{\delta \geq 0}\{A(t-\delta, t)-S(t-\delta, t)\} \leq S(t-T-\vartheta, t) .
$$

With $\sup _{\delta \geq 0}\{A(t-\delta, t)-S(t-\delta, t)\}=B(t)$ from Eq. (8) it follows for any $x$ that

$$
\sup _{t}\{\mathrm{P}[B(t) \geq x]\} \leq \sup _{t}\{\mathrm{P}[S(t-T-\vartheta, t) \geq x]\} .
$$

Since $T$ is finite and $B(t)$ is stochastically increasing there exists a finite random variable $B$ such that

$$
\lim _{t \rightarrow \infty} \mathrm{P}[B(t) \geq x]=\sup _{t}\{\mathrm{P}[B(t) \geq x]\}=\mathrm{P}[B \geq x]
$$

completing the proof of the second claim.

Example: We illustrate our estimation approach and its achievable accuracy using a computed numerical example. We consider a discrete-time system consisting of a random nonwork-conserving On-Off server. In each time slot, the server performs an independent Bernoulli trial, where it forwards one unit of data with probability $p=0.1$. This system is non-work-conserving, since, even if there is a backlog, the server remains idle with probability of $1-p$. The system is probed with constant rate probes that are each sent for a duration of 1000 time slots. Fig. 1 shows the estimated $\varepsilon$ effective service curve with $\varepsilon=10^{-3}$ computed with Th. 1 . As indicated by the thin dash-dotted lines, each probing rate $r$ contributes a linear segment with slope $r$ displaced by $-B^{\xi}(r)$ to the service curve estimate. Analytical upper and lower bounds of the service curve are included in the graph as a reference. The upper bound is computed as the amount of data forwarded in $t$ time slots with probability at least $1-\varepsilon$. By assumption, this can be computed from a binomial distribution with parameter $p$. The lower bound is computed 


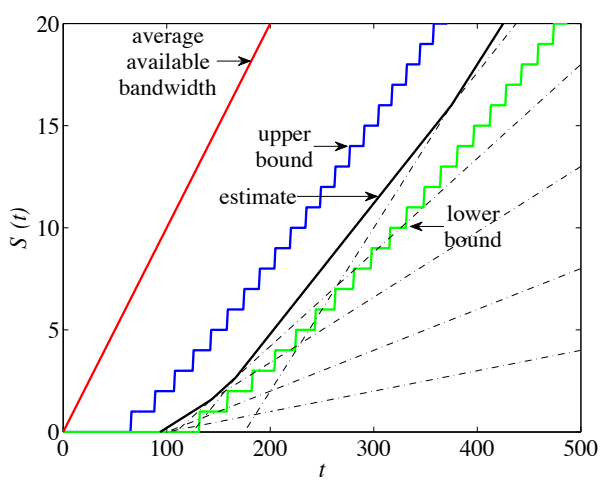

Fig. 1. Service curves of an On-Off server. The service curve estimate is composed of linear segments, that are each obtained by a single probing rate. Analytical lower and upper bounds are included for comparison. The limiting rate of the service curve estimate converges to the long-term available bandwidth. Compared to the long-term available bandwidth, the service curve provides significant details on the time scales of service availability.

from the upper bound by an application of the union bound. The depicted service curve estimate reflects a fluid flow traffic model, which is different from the staircase function obtained from the binomial distribution. Discrete-sized data packets will be incorporated into our method in Sec. III-D. For comparison, we show the service curve with a constant rate computed as the average of Eq. (1). The non-work-conserving aspect of the system is captured by the initial latency of about 100 time slots, where the estimate of $S^{\varepsilon}(t)$ in Fig. 1 evaluates to zero. While the limiting rate of the service curve estimate, defined as $\lim _{t \rightarrow \infty} \tilde{S}^{\varepsilon}(t) / t$, is equal to the long-term available bandwidth, neglecting the latency inherent to the system, the long-term available bandwidth overestimates the service in the system (by violating the upper bound). This illustrates that the $\varepsilon$-effective service curve is a non-trivial generalization of the available bandwidth concept, since the service curve can capture that the available service rate is variable at different time scales.

\section{Connection to Available Bandwidth}

In this section, we relate our stochastic system theoretic approach to the available bandwidth definition from Eq. (1). We will refer to the traffic in the system as cross traffic, and denote arrivals and departures by $A_{c}$ and $D_{c}$, respectively. Probe traffic arrivals and departures are denoted by $A_{p}$ and $D_{p}$, respectively. We will show that the service process of a work-conserving system is equivalent to the available bandwidth from Eq. (1). At the same time, we show that the commonly used probabilistic version, namely, the expected available bandwidth, necessarily leads to an overestimation of the expected departures of a system. Also, we show that a widely used definition of available bandwidth for multi-hop systems $\alpha^{n e t}(\tau, \tau+\delta)$, given in Eq. (2), can be recovered only in the limit $\delta \rightarrow \infty$.

1) Single-hop Systems: Given a work-conserving server with service process $S(\tau, t)$, where $S(\tau, t)$ denotes the amount of service available in $(\tau, t]$ [8]. For any $t \geq 0$, let $\tau \geq 0$ denote the beginning of the last busy period before $t$. From the work-conserving property it holds that $D(t)=D(\tau)+S(\tau, t)$.
Now, let $D(t)=D_{c}(t)+D_{p}(t)$ be composed of cross traffic and probe traffic, respectively. It follows that

$$
D_{p}(t)=D_{p}(\tau)+\underbrace{S(\tau, t)-D_{c}(\tau, t)}_{=: S_{l}(\tau, t)},
$$

where the service process $S_{l}(\tau, t)$ denotes the service left over by cross traffic in $(\tau, t]$. By choice of $\tau$, it holds that $D_{p}(\tau)=$ $A_{p}(\tau)$ so that $D_{p}(t)=A_{p}(\tau)+S_{l}(\tau, t)$. Given that there exists at least one $\tau \in[0, t]$ such that $D_{p}(t)=A_{p}(\tau)+S_{l}(\tau, t)$ we conclude that $D_{p}(t) \geq \inf _{\tau \in[0, t]}\left\{A_{p}(\tau)+S_{l}(\tau, t)\right\}=A_{p} \otimes$ $S_{l}(t)$. Note that since the min-plus convolution evaluates all $\tau \in[0, t]$, it is not confined to the beginning of a specific busy period. Also, from Eq. (9) it follows for all $\tau \in[0, t]$ that $D_{p}(t) \leq A_{p}(\tau)+S_{l}(\tau, t)$ since $D_{p}(\tau) \leq A_{p}(\tau)$ due to causality. It follows that $D_{p}(t) \leq \inf _{\tau \in[0, t]}\left\{A_{p}(\tau)+S_{l}(\tau, t)\right\}$. Combining the lower and the upper bound we obtain $D_{p}(t)=$ $A_{p} \otimes S_{l}(t)$, that is, $S_{l}(\tau, t)$ satisfies Eq. (5).

Next, we prove that the available bandwidth defined in Eq. (1) coincides with the leftover service process $S_{l}(\tau, t)$. Consider the stochastic leftover service process of a constant rate link $S(\tau, t)=C(t-\tau)$ that is utilized by random cross traffic with intensity $u(t)$. We obtain with Eq. (1) that

$$
\alpha(\tau, t)=\frac{\int_{\tau}^{t} C(1-u(x)) d x}{t-\tau}=\frac{S(\tau, t)-D_{c}(\tau, t)}{t-\tau},
$$

since the amount of cross traffic departures is determined by the utilization in $(\tau, t]$ as $D_{c}(\tau, t)=C \int_{\tau}^{t} u(x) d x$. It follows that

$$
\alpha(\tau, t)=\frac{S_{l}(\tau, t)}{t-\tau} .
$$

Thus, the available bandwidth from Eq. (1) for a specific time interval corresponds to the rate of the leftover service process in this time interval.

In bandwidth estimation, the time-varying nature of the available bandwidth is frequently covered by using the expected value. However, while $\alpha(\tau, t)$ can be related to the leftover service process, the following lemma states that $\mathrm{E}[\alpha(\tau, t)]$ systematically overestimates the departures.

Lemma 3: Given $D_{p}(t)=A_{p} \otimes S_{l}(t)$. It holds that

$$
\mathrm{E}\left[D_{p}(t)\right] \leq A_{p} \otimes \mathrm{E}\left[S_{l}\right](t) .
$$

Constructing examples where Lem. 3 does not hold with equality is straightforward.

Proof: Taking expectations we have

$$
\begin{aligned}
\mathrm{E}\left[D_{p}(t)\right] & =\mathrm{E}\left[\inf _{\tau \in[0, t]}\left\{A_{p}(\tau)+S_{l}(\tau, t)\right\}\right] \\
& =\sum_{\omega \in \Omega} p^{\omega} \inf _{\tau \in[0, t]}\left\{A_{p}(\tau)+S_{l}^{\omega}(\tau, t)\right\},
\end{aligned}
$$

where $S_{l}^{\Omega}$ is the sample space containing sample paths $S_{l}^{\omega}$ that occur with probability $p^{\omega}$ each. For any choice of $\tau^{\prime} \in[0, t]$,

$$
p^{\omega} \inf _{\tau \in[0, t]}\left\{A_{p}(\tau)+S_{l}^{\omega}(\tau, t)\right\} \leq p^{\omega} A_{p}\left(\tau^{\prime}\right)+p^{\omega} S_{l}^{\omega}\left(\tau^{\prime}, t\right),
$$

which yields for any $\tau^{\prime} \in[0, t]$ that

$$
\mathrm{E}\left[D_{p}(t)\right] \leq A_{p}\left(\tau^{\prime}\right)+\sum_{\omega \in \Omega} p^{\omega} S_{l}^{\omega}\left(\tau^{\prime}, t\right),
$$


since $\sum_{\omega \in \Omega} p^{\omega}=1$. It then follows that

$$
\mathrm{E}\left[D_{p}(t)\right] \leq \inf _{\tau^{\prime} \in[0, t]}\left\{A_{p}\left(\tau^{\prime}\right)+\mathrm{E}\left[S_{l}^{\omega}\left(\tau^{\prime}, t\right)\right]\right\},
$$

which completes the proof.

2) Tandem Systems: The end-to-end service process of a sequence of systems is given by the service processes of the individual systems $S^{h}(\tau, t)(h=1 \ldots H)$ as [8]

$$
S^{n e t}(\tau, t)=S^{1} \otimes \cdots \otimes S^{H}(\tau, t) .
$$

Note the difference between the min-plus convolution in Eq. (11) and the definition of end-to-end available bandwidth as a simple minimum in Eq. (2). Assuming work-conserving systems, we substitute $S^{h}(\tau, t) /(t-\tau)=\alpha^{h}(\tau, t)$ from Eq. (10). For the special case of constant rate functions $S^{h}(\tau, t) /(t-\tau)=r^{h}$, it is shown in [28] that the minplus convolution simplifies to $S^{n e t}(\tau, t) /(t-\tau)=\min _{h}\left\{r^{h}\right\}$, which coincides with the available bandwidth $\alpha^{\text {net }}(\tau, t)=$ $\min _{h}\left\{r^{h}\right\}$. Without constant rate functions, we only get $S^{n e t}(\tau, t) /(t-\tau) \leq \alpha^{n e t}(\tau, t)$. Here, we prove that equality can be recovered if the observed interval tends to infinity. We assume that the $h$-th system has a long-term average service rate $\alpha_{\infty}^{h}$, i.e., for any $\tau$, we have

$$
\liminf _{t \rightarrow \infty} \frac{S^{h}(\tau, t)}{t-\tau}=\alpha_{\infty}^{h} .
$$

Lemma 4: Given the end-to-end service process $S^{n e t}(\tau, t)$ as in Eq. (11) where the service processes of the individual systems $S^{h}(\tau, t)$ satisfy Eq. (12). For any $\tau$ it holds that

$$
\liminf _{t \rightarrow \infty} \frac{S^{n e t}(\tau, t)}{t-\tau}=\min _{h}\left\{\alpha_{\infty}^{h}\right\} .
$$

Proof: It is sufficient to show the proof for two systems. Due to the properties of the convolution, the result applies to an arbitrary number of systems by repeated application. We rewrite Eq. (11) as

$$
\frac{S^{n e t}(\tau, t)}{t-\tau}=\inf _{\theta \in[\tau, t]}\left\{\frac{S^{1}(\tau, \theta)}{t-\tau}+\frac{S^{2}(\theta, t)}{t-\tau}\right\} .
$$

Now we let $t \rightarrow \infty$. If $\alpha_{\infty}^{1}>\alpha_{\infty}^{2}$ the minimum will be attained for finite $\theta$ such that the first term of the sum goes to zero and the second term to $\alpha_{\infty}^{2}$. Otherwise, if $\alpha_{\infty}^{1}<\alpha_{\infty}^{2}$, the parameter $\theta$ will tend to $t$ such that the first term becomes $\alpha_{\infty}^{1}$ and the second term goes to zero. This gives us

$$
\liminf _{t \rightarrow \infty} \frac{S^{n e t}(\tau, t)}{t-\tau}=\min \left\{\alpha_{\infty}^{1}, \alpha_{\infty}^{2}\right\}
$$

Finally, if $\alpha_{\infty}^{1}=\alpha_{\infty}^{2}$ the result holds trivially.

\section{Max-plus Network Calculus for Timestamps}

So far, we have used the min-plus formulation of the network calculus, where arrival and departure functions $A(t)$ and $D(t)$ denote amounts of traffic in a time interval $(0, t]$. This formulation allowed us to establish the link between available bandwidth and service curves. There is an alternate (equivalent) formulation of the network calculus based on a maxplus algebra, which describes arrivals and departure functions in terms of timestamps [2], [8]. Since any probing scheme is based on taking timestamps of probing traffic, adopting a maxplus algebra for the computation of the available service is more convenient. Let $T_{A}(n)$ and $T_{D}(n)$ denote the timestamps of the $n$-th probe packet. For unit sized packets, functions $A(t)$ and $T_{A}(n)$ are related by $A(t)=\sum_{n=1}^{\infty} 1_{\left\{T_{A}(n) \leq t\right\}}$, where the indicator function $1_{\{X \leq x\}}=1$ if $X \leq x$ and zero otherwise. ${ }^{2}$

We next show how an $\varepsilon$-effective service curve can be estimated from packet timestamps, by switching to the maxplus representation of the network calculus. An additional benefit of using the max-plus representation is that it can deal with packet loss more easily, by viewing lost packets as being infinitely delayed. We next show that min-plus and max-plus representations yield identical results.

Similar to Eq. (6), an $\varepsilon$-effective service curve that operates directly on packet timestamps can be defined in the max-plus algebra as

$$
\mathrm{P}\left[T_{D}(n) \leq \max _{\nu \in[1, n]}\left\{T_{A}(\nu)+T_{\mathcal{S}}^{\varepsilon}(n-\nu)\right\}\right]>1-\varepsilon .
$$

Here, $T_{\mathcal{S}}^{\varepsilon}(n-\nu)$ specifies a shift-invariant upper bound on the amount of time required to serve $n-\nu+1$ packets. Under similar assumptions as in Th. 1, a max-plus $\varepsilon$-effective service curve can be estimated from the delay defined as $W(n)=$ $T_{D}(n)-T_{A}(n)$. Again, we use constant rate arrivals $T_{A}(n)=$ $n / r$ where $r \in R$ and let $n \rightarrow \infty$ to observe the steady state delay quantile $W^{\xi}(r)$. It can be shown that for all $n \geq 0$,

$$
T_{\mathcal{S}}^{\varepsilon}(n)=\min _{r \in R}\left\{\frac{n}{r}+W^{\xi}(r)\right\}
$$

is an $\varepsilon$-effective service curve that satisfies Eq. (13) with violation probability $\varepsilon=\sum_{r} \xi$. We provide the proof in [32] and omit it here as it closely follows the proof of Th. 1. Similar to Th. 1, Eq. (14) lays the foundation for an estimation method that, however, takes delay measurements to estimate a max-plus service curve. The relation of the two methods is established by the following theorem, which states that the max-plus service curve estimate from Eq. (14) deviates from the min-plus service curve estimate from Th. 1 by at most one packet length. The theorem uses the pseudo-inverse of service curve $T_{\mathcal{S}}$, which is defined as

$$
\left(T_{\mathcal{S}}^{\varepsilon}\right)^{-1}(t)=\sum_{n=0}^{\infty} 1_{\left\{T_{\mathcal{S}}^{\varepsilon}(n) \leq t\right\}}
$$

Theorem 2: Given a system with $\varepsilon$-effective service curves $\mathcal{S}^{\varepsilon}(t)$ from Th. 1 and $T_{\mathcal{S}}^{\varepsilon}(n)$ from Eq. (14), and assuming that the system forwards traffic in order of its arrival, the following holds:

$$
\left(T_{\mathcal{S}}^{\varepsilon}\right)^{-1}(t)-1 \leq \mathcal{S}^{\varepsilon}(t) \leq\left(T_{\mathcal{S}}^{\varepsilon}\right)^{-1}(t)
$$

The proof of Th. 2 uses the following fundamental lemma that relates the backlog of a system to its delay.

Lemma 5: Given a system with strictly increasing arrival and departure timestamps $T_{A}(n)$ and $T_{D}(n)$, respectively.

\footnotetext{
${ }^{2}$ Considering variable sized packets requires additional notation to specify packet lengths. Since many probing methods use probe packets of fixed size, the assumption of unit sized packets is justified.
} 
Assume the system serves arrivals in order. The backlog observed at the departure time $T_{D}(n)$ of packet $n$ equals

$$
\begin{aligned}
B\left(T_{D}(n)\right) & =A\left(T_{D}(n)-W(n), T_{D}(n)\right) \\
& =A\left(T_{A}(n), T_{A}(n)+W(n)\right),
\end{aligned}
$$

where $A(\tau, t)$ are the cumulative arrivals in $(\tau, t]$ and $W(n)$ is the delay of packet $n$.

Proof: From the definition of backlog we have

$$
B\left(T_{D}(n)\right)=A\left(T_{D}(n)\right)-D\left(T_{D}(n)\right) .
$$

Since the arrivals are served in order it holds that $D\left(T_{D}(n)\right)=$ $A\left(T_{A}(n)\right)$ and it follows by substitution that

$$
B\left(T_{D}(n)\right)=A\left(T_{A}(n), T_{D}(n)\right) .
$$

Using the definition of delay $W(n)=T_{D}(n)-T_{A}(n)$ completes the proof.

We use Lem. 5 to relate quantiles of the backlog and delay to each other, e.g., $B^{\xi}=r W^{\xi}$ for constant rate arrivals with rate $r$, where we denote by $B$ and $W$ the steady state backlog and delay for $n \rightarrow \infty$. We note that Little's law, i.e., $\mathrm{E}[B]=$ $\lambda \mathrm{E}[W]$ for arrivals with average rate $\lambda$, can be recovered from Lem. 5, see the technical report [31]. Equipped with Lem. 5 we now prove Th. 2.

Proof: (of Th. 2) Consider the argument of the indicator function $T_{\mathcal{S}}^{\varepsilon}(n) \leq t$ in the definition of the pseudoinverse $\left(T_{\mathcal{S}}^{\varepsilon}\right)^{-1}$. By insertion of $T_{\mathcal{S}}^{\varepsilon}(n)$ from Eq. (14) we have $\min _{r \in R}\left\{n / r+W^{\xi}(r)\right\} \leq t$ and after some reordering

$$
n \leq \max _{r \in R}\left\{r t-r W^{\xi}(r)\right\} .
$$

Instantiating Lem. 5 with $A(t)=\lfloor r t\rfloor$ yields the backlog $B\left(T_{D}(n)\right)=\lfloor r W(r, n)\rfloor$. Letting $n \rightarrow \infty$ and taking quantiles we obtain $B^{\xi}(r)=\left\lfloor r W^{\xi}(r)\right\rfloor=r W^{\xi}(r)-\vartheta(r)$, where $\vartheta(r) \in[0,1)$. It follows that the condition $T_{\mathcal{S}}^{\varepsilon}(n) \leq t$ is equivalent to

$$
n \leq \max _{r \in R}\left\{r t-B^{\xi}(r)-\vartheta(r)\right\} .
$$

With Th. 1 and since $n=0,1,2, \ldots$ is an integer, the claim follows.

\section{Derivation of the Probing Methodology}

We next address how the results from Sec. III can be developed into practical probing schemes for networks with random service. The probing schemes are expressed as described in Sec. III-D, where arrival and departure timestamps of packet train probes are used to measure steady state delay quantiles, and to compute an estimate of the service curve.

We describe a measurement experiment with constant rate packet train probes by a tuple $\langle R, N, I\rangle$, where $R$ is the set of probing rates, $N$ is the number of packets in a train, and $I$ is the number of repeated measurements. The selection of these parameters is crucial for a probing scheme, yet, the derivations in the previous section do not provide guidelines for selecting values. For example, a single reading of the steady state delay $W(r)$ from a packet train is ideally taken from an infinitely long packet train $(N \rightarrow \infty)$. Also, computing the exact tail distribution of the delay requires an infinite
Fig. 2. Multi-hop network with multiple 100 Mbps bottleneck links.

number of repeated measurements $(I \rightarrow \infty)$. Clearly, such ideal measurement experiments are not viable. In this section, we will develop and evaluate guidelines for practical choices for $\langle R, N, I\rangle$. In Sec. IV-A, we discuss the selection of the rates. In Sec. IV-B, we show how to obtain stationary delay estimates using finite packet trains and a finite number of repeated measurements with the usage of statistical methods. In Sec. IV-C, we discuss how to extract information from short packet trains. In Sec. IV-D, we apply our method to non-linear and lossy networks.

For an experimental evaluation we resort to a local testbed as well as the Emulab testbed [42], which offer controlled experiments on real networking equipment. We consider the topology shown in Fig. 2, where probe traffic and cross traffic are multiplexed at a tandem of 100 Mbps bottleneck links. The capacities and delays in the network are specified in the figure. We consider different packet schedulers, including priority, fair queueing, and FIFO, and different buffer sizes at the bottleneck links. The default configuration is a network with a single bottleneck link and priority scheduling with high priority to cross traffic, and a large buffer size (of $10^{6}$ packets). Cross traffic has a mean rate of $50 \mathrm{Mbps}$, and consists of equally spaced packet bursts of back-to-back packets whose size follows a truncated Exponential or Pareto distribution. A comparison of outcomes for these distributions reveals the sensitivity of the measurement methodology to the burstiness of network traffic. The average size of a packet burst is 1500 Byte and the shape parameter of the Pareto distribution is 1.5 . With the chosen link capacity and cross traffic rate, the limiting rate of the service process (= longterm available bandwidth) is 50 Mbps. We use D-ITG [4] to generate cross traffic. D-ITG generates truncated arrival distributions to conform to the maximum IP payload size of 64 kByte. The payload is further divided by IP fragmentation to packet sizes of at most 1500 Byte. For probe traffic, we use Rude/Crude [25] which emits constant bit rate packet trains consisting of packets with a size of 1500 Byte. NTP is used for time synchronization. For the impact of asynchronous clocks, we refer to [28].

\section{A. Selection of Probing Rates}

The selection of the set of probing rates presents a tradeoff. On the one hand, adding probing rates improves the estimate of the $\varepsilon$-effective service curves, since each rate contributes an additional linear segment (see Fig. 1). On the other hand, since we compute the violation probability by an application of the union bound, adding probing rates increases the violation probability. We use an algorithm that seeks to find a small set of characteristic rates that contribute to the service curve. The algorithm combines a binary increase and a binary search algorithm, similar to the rate selection procedure in [17].

The algorithm has as parameter $r_{a c c}$ to specify the desired rate resolution. The binary increase starts at $r_{1}=r_{a c c}$. As long as the probes at rate $r_{i}$ measure a finite delay quantile, the rate is doubled. The estimation of the delay quantile is explained in Sec. IV-B. The first rate $r_{i}$ at which no finite delay quantile 
can be measured is used to start a binary search in the interval $\left[r_{i-1}, r_{i}\right]$ using the same test criterion. Each additional probing rate halves the interval. Once the interval achieves the target accuracy $r_{a c c}$ the rate scan is terminated. Let $\tilde{r}$ be the largest rate that achieves a finite delay quantile. As an example if $\tilde{r}=50 \mathrm{Mbps}$ and $r_{a c c}=4 \mathrm{Mbps}$ the algorithm probes at rates $r_{i}=4,8,16,32,64,48,56$, and $52 \mathrm{Mbps}$ and stops at the interval $[48,52]$.

The total number of rates probed by the binary increase/binary search algorithm is $2\left\lfloor\log _{2}\left(\tilde{r} / r_{a c c}\right)\right\rfloor+2$, where the binary increase algorithm requires $\left\lfloor\log _{2}\left(\tilde{r} / r_{a c c}\right)\right\rfloor+2$ steps until it first exceeds $\tilde{r}$, and the binary search performs another $\left\lfloor\log _{2}\left(\tilde{r} / r_{a c c}\right)\right\rfloor$ steps to ensure the target accuracy.

\section{B. Estimation of Steady State Delays}

Next, we discuss how to estimate the tail distribution of steady state delays using finite length packet train probes. We employ a statistical test to detect stationarity of a time series, and use it to adapt the length of packet train probes to the variability of cross traffic.

1) Stationarity Test: By definition, Eq. (14) uses steady state delays to compute a service curve. While Lem. 2 states that the steady state delay distribution exists as long as the rate of probe arrivals does not exceed the limiting rate of the service process, reaching the steady state requires infinitely long packet trains. To determine the steady state delay from a finite length packet train, we use a statistical test that detects if the delays $W(n)$ of a sequence of probe packets $n \in[0, N-1]$ satisfy stationarity. If stationarity is detected, we use the delay of the last packet of a packet train as an estimate of the steady state delay. The delay values of all other packets from the same packet train are discarded due to possible correlations. If stationarity cannot be detected, we set the delay estimate to infinity. We repeat the measurement $I$ times for each probing rate $r$ to measure the $(1-\xi)$-quantile of the delay $W^{\xi}(r)$. Note that due to the minimum in Eq. (14), delay quantiles of infinity do not contribute to the service curve estimate.

To detect stationarity of the delay series observed by a packet train, we use the unit root test from Elliot, Rothenberg and Stock (ERS) [12]. The ERS test is based on an autoregressive moving average model. The null hypothesis of the test is that the data series has a unit root, which implies nonstationarity. The ERS test returns a single value referred to as ERS statistic. If the ERS statistic falls below a critical value, the null hypothesis is rejected and stationarity is assumed.

2) Adaptive Train Length: Since the minimal train length that permits detecting stationarity is not known a priori, we define a procedure that adaptively increases the train length. When the ERS test indicates non-stationarity for a share of more than $\xi$ of the packet trains sent at a certain rate, then either the stationary $(1-\xi)$-delay quantiles cannot be achieved at this probing rate, or the train length is too short to reliably detect stationarity. To assess whether increasing the train length can help, we inspect the trend of the ERS statistic. We compute the ERS statistic for the first half of the train and for the entire train. If the ERS statistic decreases, i.e., if the ERS statistic of the first half of the train is larger than the ERS statistic of the entire train, we interpret this as an indication

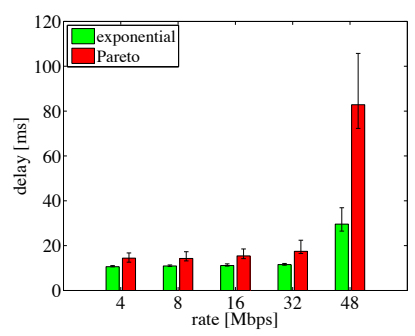

(a) (b) Stationary 0.95-delay quantile Re-

quired train

length

Fig. 3. Fig. 3(a) shows the required train length needed to observe stationary delays at a link with Exponential and Pareto cross traffic, and a limiting rate of 50 Mbps. For the train lengths in Fig. 3(a), Fig. 3(b) presents the observed delay values as stationary 0.95 -delay quantiles (with 0.95 confidence intervals).

that longer trains may achieve stationarity. We refer to this test as the trend test that is passed by a packet train if its ERS statistic decreases. If the majority of the packet trains sent at a certain rate passes the trend test, we double the train length and carry out the measurements at this rate anew. We repeat this procedure until either stationarity is achieved or the majority of the trains fails the trend test.

We next show the train length required to achieve stationarity at a certain probing rate. Fig. 3(a) shows the train length that permits detecting stationarity for a share of at least $(1-\xi)=0.95$ of $I=250$ packet trains sent at different probing rates and for different types of cross traffic each. The probing rates are chosen according to the algorithm from Sec. IV-A and the train length is adapted as described above starting at a minimum train length of 100 packets. The results show that the required train length is sensitive to the distribution of cross traffic and to the probing rate. The required train length increases sharply, when the probing rate approaches the limiting rate of $50 \mathrm{Mbps}$.

3) Tail Distribution: The computation of $\varepsilon$-effective service curves requires the $(1-\xi)$-quantiles of the stationary delays, $\widetilde{W}^{\xi}(r)$. Since each packet train only provides one reading of the stationary delays, obtaining a delay quantile requires multiple packet trains for each probing rate. In particular, to compute a 0.95 -delay quantile, the minimum number of repeated measurements for a given rate is $I=20$.

The delays observed by different packet trains can be assumed to be independent when packet trains have random start times (see [3] for a discussion). We can quantify the accuracy of the delay quantiles using confidence intervals (which, for quantiles, are computed from the binomial distribution). This allows us to increase the number of packet trains until a desired accuracy is met. Fig. 3(b) displays the stationary 0.95delay quantiles and corresponding 0.95 confidence intervals achieved with $I=250$ repeated measurements (where packet train lengths are given in Fig. 3(a)). The indicated confidence intervals show that the accuracy decreases when the probing rate approaches the limiting rate and when cross traffic is more bursty. To reduce the number of repeated measurements, we refer to the technical report [31] for methods to predict the tail of the delay distribution. 


\section{Short Packet Train Probes}

As seen in Fig. 3(a), when the network is close to saturated, measuring stationary delays may require very long packet trains. In this section, we discuss how the limiting rate and a service curve can be estimated with small sized packet trains, without requiring stationary delays.

1) Limiting Rate Estimation: In principle, the limiting rate of a service curve estimate is the largest probing rate that observes finite steady state delays. In practice, however, the required packet train length needed to read steady state delays may become prohibitive. Thus, we abandon trying to read steady state delays in this regime, and, instead, estimate the limiting rate directly. Based on the ERS test and the trend test, we devise a heuristic method to obtain the limiting rate with packet trains with at most $N$ packets.

In Sec. IV-B2, we used the ERS test to detect stationarity of delays, and the trend test to indicate that stationarity may be observable for longer packet trains (sent at the same rate). To limit the train length at $N$, we now consider passing the trend test as sufficient to assume that a finite steady state delay exists at a given rate. When the trend test fails, we assume that no finite steady state delay exists. We use the interpretation of the trend test, i.e., finite steady state delays exist or do not exist, as criteria in the binary increase/binary search algorithm from Sec. IV-A for increasing or decreasing the probing rate. Then, the largest probing rate for which the trend test passes is an estimate of the limiting rate.

In Fig. 4, we evaluate the impact of the train length on the fidelity of the trend test. We use again the network shown in Fig. 2. Since the network has a limiting rate of $50 \mathrm{Mbps}$, packet trains should pass the trend test if the probing rate is below $50 \mathrm{Mbps}$, and fail the trend test if the probing rate exceeds $50 \mathrm{Mbps}$. The bars in Fig. 4 depict the fraction of trains that pass the trend test out of a total of $I=1000$ trains, for probing rates $r=40-60 \mathrm{Mbps}$. We show results for packet trains with a length of $N=50-800$. The figure shows that the likelihood of a correct classification increases when the train length is increased, and that it decreases when the probing rate approaches the limiting rate, and when the cross traffic is more bursty. On the other hand, note that the majority of trains makes a correct classification.

We can exploit the last observation to achieve a robust classification when the number of packet trains is small. We perform measurements of $I$ packet trains, perform the trend test for each train, and then conduct a majority decision. By selecting $I$ to be an odd number, we can ensure that that a majority decision is always feasible. Assuming independence of packet trains, we compute the probability that a majority of trains passes the test from Fig. 4 via the binomial distribution. Fig. 5 shows the results for a train length of $N=200$ packets and $I=11-51$ repeated measurements. Even for Pareto traffic, using a majority decision of the trend tests results in a correct classification.

2) Service Curve Estimation: Short packet trains often do not pass the ERS test, and, therefore, do not allow a reading of stationary delays. It is possible to construct a service curve with non-stationary delays, which, however, is only valid for time intervals with limited range. Using the procedure from

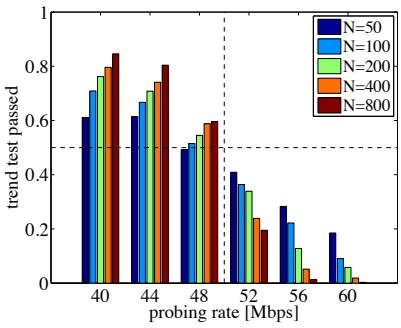

(a) Exponential cross traffic (b)

Pareto

cross

traf-

fic
Fig. 4. Fraction of packet trains, out of $I=1000$ trains, that pass the trend test for different probing rates and given packet train length $N=50-800$. Packet trains with a rate below the limiting rate of $50 \mathrm{Mbps}$ are classified correctly if they pass the trend test, and packet trains with a higher rate are classified correctly if they fail the trend test.

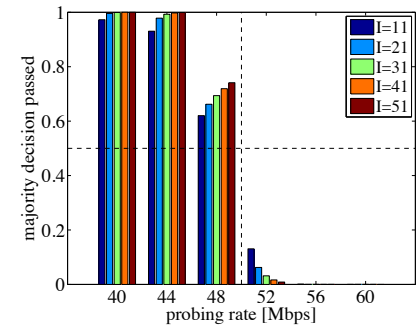

(a) Exponential cross traffic

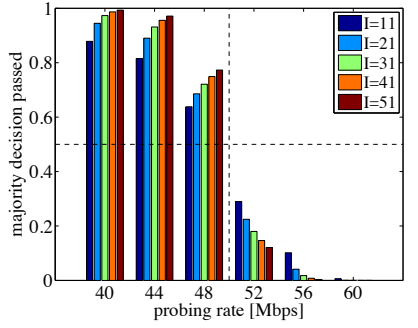

(b) Pareto cross traffic
Fig. 5. Outcome of a majority decision on the trend test using $I$ repeated measurements for a train length of $N=200$ packets. As in Fig. 4, trains with a rate below $50 \mathrm{Mbps}$ should pass the trend test, and packet trains with a rate above 50 Mbps should fail the trend test.

Sec. IV-C we can measure the delay of the last packet (with index $N$ ) of each train and compute a service curve estimate $T_{S}^{\varepsilon}(n)$ with Eq. (14) for the range $n \in[0, N-1]$. The details are described in the technical report [31]. By selecting $N$ sufficiently large, the range of validity of the service curve can be made large enough to cover the time scales of interest.

For the same network as before, Fig. 6 depicts service curves obtained with packet trains of length $N=800-12800$, and compares them to a service curve estimate with unrestricted train lengths (which observes stationary delays). The probing rates are set for a target resolution of $r_{a c c}=4 \mathrm{Mbps}$ and the number of repeated measurements is $I=250$. Fig. 6 shows the computed service curves. In the figure, the limiting rate of $50 \mathrm{Mbps}$ is indicated by the dashed diagonal lines. It is evident that all service curve estimates closely track the limiting rate of $50 \mathrm{Mbps}$. For Exponential cross traffic, service curves computed with $N=800$ provide similar results as unrestricted packet trains. For the burstier Pareto traffic, we observe that the service curve segments computed by short trains sometimes overestimate the service curve estimate obtained from stationary delays. Here, when the probing rate approaches the limiting rate, the delay estimates obtained from short trains underestimate the stationary delay distribution, which results in an overestimation of the service curve.

\section{Non-linear and Lossy Networks and Elastic Cross Traffic}

So far, we assumed that network elements such as links, queues, and schedulers can be modeled by Eq. (5) as lossless 

(a) (b)
Ex-Pareto
po-cross
nentraf-
tialfic
cross
traf-
fic

Fig. 6. Service curve estimates from trains of length $N$ compared to unrestricted trains that observe stationary delays.

linear systems. Next, we show results for systems where these assumptions are relaxed.

A FIFO scheduler is a prime example of a non-linear system. As shown in [13], a FIFO scheduler operates as a linear system only when the traffic (from probe and cross traffic) is less than the capacity of the system. When it becomes overloaded, the FIFO scheduler enters into a nonlinear regime. Thus, the application of linear system theory to FIFO schedulers is justified only when the capacity is not saturated. In our case, since the stationarity test ensures that the probing traffic does, on average, not exhaust the capacity of the network, the linearity assumption is often justified. Bursty arrivals, however, cause short term violations of linearity.

The assumption of lossless systems can be easily relaxed in a max-plus algebra, by simply modeling a dropped packet as incurring an infinite delay. That is, if packet $n$ has been dropped, we set $T_{D}(n)=\infty$. As a result, probing rates experiencing a packet loss ratio of $\xi$ or more do not contribute to the service curve estimate.

In Fig. 7 we show service curve estimates obtained for a link with fair and priority scheduling, both of which are linear, to FIFO scheduling, which is non-linear in overload. For priority scheduling, we assume that cross traffic has a higher priority. Buffers are large ( $10^{6}$ packets) enough so that no packet losses occur. For a FIFO scheduler, we also include results with a small buffer size of 200 packets, which results in moderate packet losses (The loss is less than $1 \%$ at the limiting rate). The probing parameters are $r_{a c c}=4 \mathrm{Mbps}, I=250, \xi=0.05$, and $N=800$. For all scenarios, the service curves exhibit a latency of slightly above $10 \mathrm{~ms}$, which matches the propagation delay of the bottleneck link (see Fig. 2). The service curve estimate for the fair scheduler is a straight line since the scheduler allocates a fair share of $50 \mathrm{Mbps}$ to the probe traffic regardless of the burstiness of cross traffic. The service curve estimate at a priority scheduler, on the other hand, is sensitive to the type of cross traffic. Here, the higher priority cross traffic results in additional latencies and a lower initial rate of the service curve. For the FIFO scheduler, we observe that the service curve estimates with small buffers and large buffers are very close, indicating that our estimation method deals well with packet losses. For Pareto cross traffic and with small buffers, the additional packet losses compared to Exponential cross traffic result in a larger estimate of the service curve.

We also evaluated the estimation method in a network where cross traffic consists of elastic TCP traffic using the TCP Cubic algorithm. Since TCP reacts to congestion in the network, probing traffic could displace TCP traffic and overestimate the available bandwidth. We compare service curve estimates made with 1 UDP cross traffic flow to estimates with 1, 10,

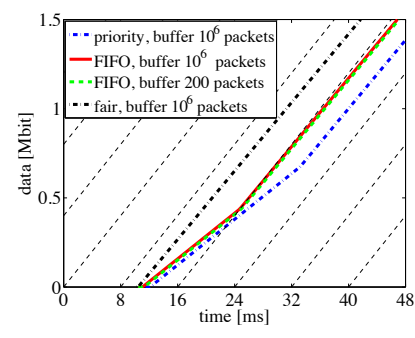

(a) Exponential cross traffic

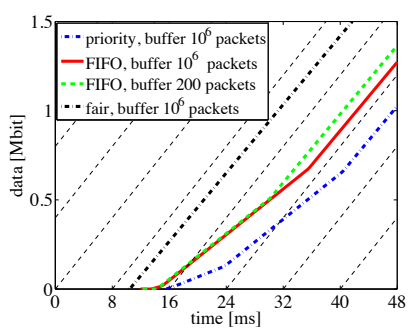

(b) Pareto cross traffic
Fig. 7. Service curve estimates for priority, fair, and FIFO scheduling with large and small buffer.

TABLE I

LIMITING RATE ESTIMATES WITH ELASTIC CROSS TRAFFIC.

\begin{tabular}{|c|c|c|c|c|}
\hline \multirow{2}{*}{$\begin{array}{l}\text { cross } \\
\text { traffic } \\
\text { flows }\end{array}$} & \multicolumn{2}{|c|}{ exponential } & \multicolumn{2}{|c|}{ Pareto } \\
\hline & $\begin{array}{c}\text { limiting rate } \\
{[\mathrm{Mbps}]}\end{array}$ & $\begin{array}{c}\text { latency } \\
{[\mathrm{ms}]}\end{array}$ & $\begin{array}{c}\text { limiting rate } \\
{[\mathrm{Mbps}]}\end{array}$ & $\begin{array}{c}\text { latency } \\
{[\mathrm{ms}]}\end{array}$ \\
\hline $1 \mathrm{TCP}$ & 48 & 15 & 48 & 22 \\
\hline 10 TCP & 48 & 16 & 48 & 23 \\
\hline $100 \mathrm{TCP}$ & 48 & 16 & 48 & 24 \\
\hline 1 UDP & 48 & 19 & 48 & 26 \\
\hline
\end{tabular}

and 100 TCP cross traffic flows. In each case, the average rate of cross traffic is $50 \mathrm{Mbps}$, using the same parameters as before $\left(r_{a c c}=4 \mathrm{Mbps}, I=250, \xi=0.05, N=800\right)$. The scheduling is FIFO with a buffer size of $10^{6}$ packets. Traffic generation at the application follows an Exponential or Pareto distribution. Tab. I depicts the initial latency and the limiting rate of the computed service curve (This corresponds to expressing the service curve to a simple latency-rate function). For all cases, the same limiting rate of $48 \mathrm{Mbps}$ is computed. Also, the estimates of the latencies depend only on the traffic distribution (Pareto or Exponential), but not on the composition of the traffic. This indicates that TCP traffic is not displaced by the estimation method.

\section{Comparative Evaluation}

Here, we compare the stochastic bandwidth estimation method with bandwidth estimation methods from the literature. We consider the network topology in Fig. 2, with FIFO scheduling, and parameters as discussed in Sec. IV. We also present measurements of an IEEE 802.11a network.

\section{A. Service Curve Estimation}

We first perform a comparison with a probing method for min-plus time-invariant deterministic linear systems from [28], for the network in Fig. 2 with a single bottleneck with FIFO scheduling and a buffer size of $10^{6}$ packets. The method uses constant rate packet trains with fixed length ( $N=800$ packets), where the rate of the packet trains is incremented between successive trains by $8 \mathrm{Mbps}$. We match these parameters for the stochastic bandwidth estimation, by using adaptively varied train length with up to 800 packets and a target accuracy of $r_{a c c}=8 \mathrm{Mbps}$. Also, we set $\xi=0.05$. For both methods we present results from $I=200$ iterations. The stochastic bandwidth estimation uses repeated measurements to obtain $(1-\xi)$-delay quantiles, as well as 0.95 confidence intervals of the delays to derive a single estimate of an $\varepsilon$ effective service curve. Since the method for deterministic 


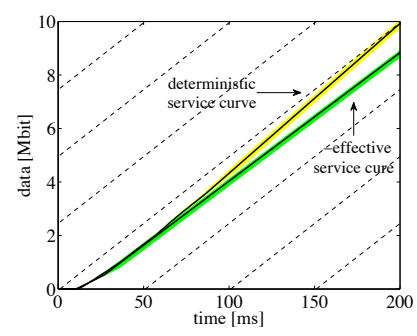

(a) Exponential cross traffic

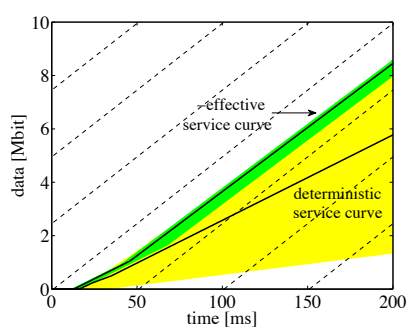

(b) Pareto cross traffic
Fig. 8. Comparison of stochastic bandwidth estimation and deterministic bandwidth estimation. The $\varepsilon$-effective service curve is compared to the average deterministic service curve, with 0.95 confidence intervals indicated by shaded areas.

time-invariant systems [28] generates one deterministic service curve estimate in each iteration, we compute the mean and 0.95 confidence intervals for all iterations.

Fig. 8 shows the average value of the deterministic service curve and the $\varepsilon$-effective service curve. Confidence intervals are shown as shaded areas. With Exponential cross traffic both deterministic and stochastic service curve estimates give comparable results. A closer comparison with the diagonal reference lines shows that the deterministic service curve overestimates the limiting rate of $50 \mathrm{Mbps}$. For Pareto cross traffic, the deterministic service curve results in a lower estimate, and very large confidence intervals. The $\varepsilon$-effective service curve recovers the limiting rate closely with small confidence intervals. Note that the service curves capture the non-work conserving aspect of the available service in terms of the initial latency.

\section{B. Bandwidth Estimation Tools}

Most existing bandwidth estimation methods seek to find the long-term available bandwidth as defined in Section II. We present an example to illustrates that the long-term available bandwidth only presents part of the information on the available service in a network.

We consider a single 100 Mbps FIFO bottleneck link with Exponential cross traffic sent at rate $50 \mathrm{Mbps}$. We set the buffer limit to 200 packets (experiments with larger buffers yielded similar results). The other network and probing parameters are as in Sec. IV-D.

For the example, we select the Pathload [17] tool, which is frequently used as a benchmark method. The method reports a lower and an upper bound of the available bandwidth. (We refer to our technical report for a comparison with IGI/PTR [16], Spruce [41], and dietTOPP [22]). As a reference, we also include an analytically computed $\varepsilon$-effective service curve, which is computed as a leftover service curve [7] using a sample path bound for the cross traffic. For Exponential cross traffic, the analytical curve is computed with the Erlang distribution and the union bound.

Fig. 9 presents the results. Since the available bandwidth in Eq. (1) is defined as a rate, we convert the estimated and analytical $\varepsilon$-effective service curves $\mathcal{S}^{\varepsilon}(t)$ to a rate function $\mathcal{S}^{\varepsilon}(t) / t$. We note that the estimated $\varepsilon$-effective service curve matches the analytical reference very closely. The gray area shows the median of 100 repeated Pathload measurements.

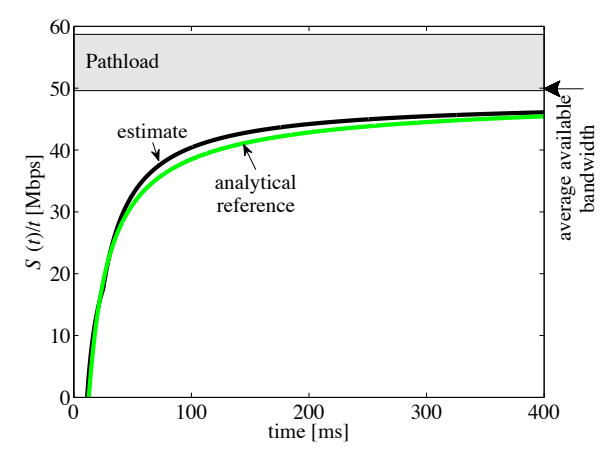

Fig. 9. Comparison of estimated $\varepsilon$-effective service curve with analytical reference of $\varepsilon$-effective service curve and estimates by Pathload tool (Service curves are displayed as rate functions to allow for comparison).

The figure shows that Pathload recovers the long-term available bandwidth well. On the other hand, the analytical and estimated $\varepsilon$-effective service curves express that significantly fewer resources are available on shorter time scales, which is due to the short term randomness of cross traffic and link delays.

\section{Multiple Bottleneck Links}

We now address networks with multiple bottleneck links. In such networks, many estimation methods have been reported to severely underestimate the long-term available bandwidth on an end-to-end path [19]. In Sec. III-C, we showed that the end-to-end available bandwidth from Eq. (2) is generally greater than the end-to-end service process of a network given by Eq. (11). It recovers the long-term available bandwidth if the observation duration tends to infinity.

Since these results suggest that longer packet trains provide better estimates, we investigate how the length of packet trains impacts the accuracy of end-to-end available bandwidth estimates. We use the topology in Fig. 2, with one, three, and five $100 \mathrm{Mbps}$ bottleneck links with FIFO scheduling and large buffers (of $10^{6}$ packets). At each bottleneck link, we have independent Exponential cross traffic with an average rate of $50 \mathrm{Mbps}$. The remaining parameters are as defined at the end of Sec. V-B. We report results of the limiting rate for the $\varepsilon$-effective service curve estimate from Sec. IV-C with a target accuracy of $r_{a c c}=1 \mathrm{Mbps}$, and the available bandwidth range reported by Pathload, where we show the median of 100 trials. We vary the maximum length of a packet train from 100 to 1600 packets. Since Pathload by default uses packet trains with a fixed length of 100 packets, we modified the source code of the tool.

Tab. II shows the limiting rates of the $\varepsilon$-effective service curve estimates and the available bandwidth bounds computed by Pathload. With short packet trains, estimates for a single bottleneck link are accurate. However, increasing the number of bottleneck links results in lower estimates. Longer packet trains provide better results for multiple bottleneck links, with both compared methods. This leaves open the possibility that underestimation of the long-term available bandwidth can be remedied by increasing the length of packet train probes. 
TABLE II

AVAILABLE BANDWIDTH ESTIMATES FOR MULTIPLE BOTTLENECK LINKS

\begin{tabular}{|c|c|c|c|c|c|c|c|c|c|}
\hline \multirow{4}{*}{$\begin{array}{c}\text { train } \\
\text { length } \\
\text { [packets] }\end{array}$} & \multirow{2}{*}{\multicolumn{3}{|c|}{$\begin{array}{c}\varepsilon \text {-eff. serv. curve } \\
\text { (limiting rate) } \\
{[\mathrm{Mbps}]}\end{array}$}} & \multicolumn{6}{|c|}{$\begin{array}{c}\text { Pathload } \\
\text { (available bandwidth) }[\mathrm{Mbps}]\end{array}$} \\
\hline & & & & \multirow{2}{*}{\multicolumn{3}{|c|}{$\begin{array}{l}\text { lower bound } \\
\text { bottleneck links }\end{array}$}} & \multirow{2}{*}{\multicolumn{3}{|c|}{$\begin{array}{c}\text { upper bound } \\
\text { bottleneck links }\end{array}$}} \\
\hline & & enec & links & & & & & & \\
\hline & 1 & 3 & 5 & 1 & 3 & 5 & 1 & 3 & 5 \\
\hline 100 & 48 & 43 & 41 & 50 & 43 & 38 & 58 & 53 & 44 \\
\hline 200 & 48 & 45 & 44 & 51 & 45 & 41 & 54 & 52 & 45 \\
\hline 400 & 48 & 47 & 46 & 51 & 47 & 43 & 53 & 51 & 45 \\
\hline 800 & 48 & 47 & 47 & 51 & 49 & 45 & 52 & 51 & 46 \\
\hline 1600 & 48 & 48 & 48 & 51 & 49 & 46 & 51 & 51 & 47 \\
\hline
\end{tabular}

Fig. 10. Wireless network with several cross traffic senders.

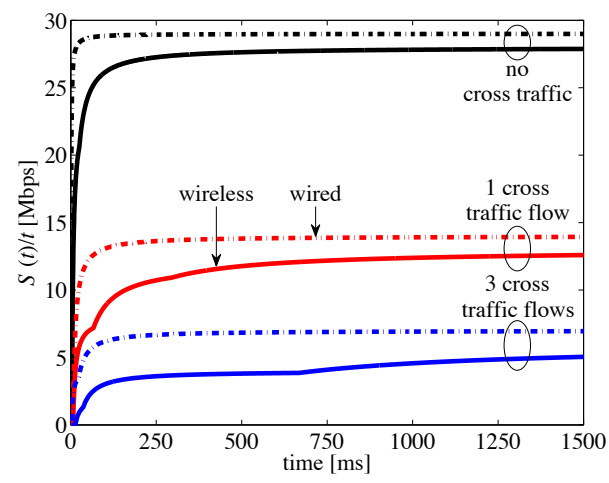

Fig. 11. Service curves as rate functions for a wireless IEEE 802.11a network, and a wireline $30 \mathrm{Mbps}$ link with fair queueing. The graphs show the rate of the service curve estimates with 0,1 , and 3 cross traffic flows.

\section{IEEE 802.11a WiFi Networks}

We apply the stochastic bandwidth estimation to a WiFi network, where the medium access control of the DCF results in a non-deterministic and non-work-conserving channel allocation. ${ }^{3}$ We measure an IEEE 802.11a testbed network with and without cross traffic as shown in Fig. 10. Probe and cross traffic compete for the wireless medium using the DCF. All transmitted packets are received by an access point, which forwards the packet to a receiver station on a wireline 100 Mbps link. Since the DCF has been previously related to a fair queueing system [6], we compare the results to a wired network that employs fair queueing at a bottleneck link. The capacity of the wired bottleneck link is throttled to $30 \mathrm{Mbps}$. This corresponds to the maximum achievable throughput in the WiFi 802.11a network [5]. As we seek to present results on the DCF, we avoid additional randomness by using constant rate cross traffic with a rate of $25 \mathrm{Mbps}$. The rate is divided equally among the cross traffic flows, so that each flow uses at least its fair share. This allows exploring the behavior of the DCF in a fully utilized IEEE 802.11a network. The parameters for the packet trains are $N=800$ packets, $I=250$ iterations, $\xi=0.05$, and a target accuracy of $r_{a c c}=1 \mathrm{Mbps}$. Fig. 11 shows the rate of the service curve estimates $\mathcal{S}^{\varepsilon}(t) / t$ for the WiFi network and the wireline fair queueing link. For the fair scheduler, without cross traffic, the rate quickly approaches the bound

\footnotetext{
${ }^{3}$ We note that [40] recently applied our method to compute service curves of a simulated wireless channel.
}

of $30 \mathrm{Mbps}$. When cross traffic is added, the rates track the fair share of 15 and $7.5 \mathrm{Mbps}$ for 1 and 3 cross traffic flows, respectively. For the WiFi network, the limiting rates estimate do not quite reach the computed fair share. Also, the slower convergence to the limiting rate reflects additional delays in the WiFi network. The lower rate and higher delays seen in the WiFi network are due to the accumulated impacts of the random backoff of the DCF, collisions, and retransmissions.

\section{CONCLUSION}

We have presented a system-theoretic foundation for bandwidth estimation of networks with random service, where we used the framework of the stochastic network calculus to derive a method that estimates an $\varepsilon$-effective service curve from steady state backlog or delay quantiles observed by packet train probes. The service curve model extends to networks of nodes, as well as to non-work-conserving systems. The $\varepsilon$-effective service curve characterizes service availability at different time scales, and recovers the long-term available bandwidth as its limiting rate. While ideal measurements require an infinite repetition of infinitely long packet trains, we showed that practical estimation methods can be achieved by applying statistical tests and using appropriate heuristics. We found that cross traffic variability, the number of bottleneck links, and the target accuracy of the estimate have a significant impact on the amount of probes required. We presented measurement examples, which showed that estimates of $\varepsilon$ effective service curves can disclose essential characteristics of random service in wired and wireless networks, which are not reflected in the long-term available bandwidth.

\section{REFERENCES}

[1] F. Agharebparast and V. C. M. Leung. Slope domain modeling and analysis of data communication networks: A network calculus complement. In Proc. IEEE ICC, pages 591-596, June 2006.

[2] F. Baccelli, G. Cohen, G. J. Olsder, and J.-P. Quadrat. Synchronization and Linearity: An Algebra for Discrete Event Systems. John Wiley \& Sons Ltd., 1992.

[3] F. Baccelli, S. Machiraju, D. Veitch, and J. Bolot. The role of PASTA in network measurement. In Proc. ACM SIGCOMM, pages 231-242, Sept. 2006.

[4] A. Botta, A. Dainotti, and A. Pescape. Multi-protocol and multi-platform traffic generation and measurement. In Proc. IEEE INFOCOM Demo, May 2007.

[5] M. Bredel and M. Fidler. A measurement study of bandwidth estimation in IEEE $802.11 \mathrm{~g}$ wireless LANs using the DCF. In Proc. IFIP Networking, pages 314-325, May 2008.

[6] M. Bredel and M. Fidler. Understanding fairness and its impact on quality of service in IEEE 802.11. In Proc. IEEE INFOCOM, pages 1098-1106, Apr. 2009.

[7] A. Burchard, J. Liebeherr, and S. Patek. A min-plus calculus for end-toend statistical service guarantees. IEEE Trans. Inf. Theory, 52(9):41054114, Sept. 2006.

[8] C.-S. Chang. Performance Guarantees in Communication Networks. Springer-Verlag, 2000.

[9] A. Delphinanto, T. Koonen, and F. den Hartog. Real-time probing of available bandwidth in home networks. IEEE Commun. Mag., 49(6):134-140, June 2011.

[10] B. K. Dey, D. Manjunath, and S. Chakraborty. Estimating network link characteristics using packet-pair dispersion: A discrete-time queueing theoretic analysis. Computer Networks, 55:1052-1068, April 2011.

[11] S. Ekelin et al. Real-time measurement of end-to-end available bandwidth using Kalman filtering. In Proc. IEEE/IFIP NOMS, pages 73-84, Apr. 2006.

[12] G. Elliott, T. J. Rothenberg, and J. H. Stock. Efficient tests for an autoregressive unit root. Econometrica, 64(4):813-836, July 1996. 
[13] Y. Ghiassi-Farrokhfal and J. Liebeherr. Output characterization of constant-bit-rate traffic in FIFO networks. IEEE Commun. Lett., 13(8):618-620, Aug. 2009.

[14] P. Hága, K. Diriczi, G. Vattay, and I. Csabai. Understanding packet pair separation beyond the fluid model: The key role of traffic granularity. In Proc. IEEE INFOCOM, pages 2374-2386, Apr. 2006.

[15] T. Hisakado, K. Okumura, V. Vukadinovic, and L. Trajkovic. Characterization of a simple communication network using Legendre transform. In Proc. IEEE ISCAS, pages 738-741, May 2003.

[16] N. Hu and P. Steenkiste. Evaluation and characterization of available bandwidth probing techniques. IEEE J. Sel. Areas Commun., 21(6):879894, Aug. 2003.

[17] M. Jain and C. Dovrolis. Pathload: A measurement tool for end-to-end available bandwidth. In Proc. PAM, pages 14-25, Mar. 2002.

[18] M. Jain and C. Dovrolis. End-to-end available bandwidth: measurement methodology, dynamics, and relation with TCP throughput. IEEE/ACM Trans. Netw., 11(4):537-549, 2003.

[19] M. Jain and C. Dovrolis. Ten fallacies and pitfalls on end-to-end available bandwidth estimation. In Proc. ACM IMC, pages 272-277, Oct. 2004.

[20] Y. Jiang and Y. Liu. Stochastic Network Calculus. Springer, 2008.

[21] A. Johnsson and M. Bjorkman. On measuring available bandwidth in wireless networks. In Proc. IEEE LCN, pages 861-868, Oct. 2008.

[22] A. Johnsson, B. Melander, and M. Björkman. Bandwidth measurement in wireless networks. In Proc. Med-Hoc-Net, pages 89-98, June 2005.

[23] P. Kanuparthy, C. Dovrolis, K. Papagiannaki, S. Seshan, and P. Steenkiste. Can user-level probing detect and diagnose common home-wlan pathologies. ACM SIGCOMM CCR, 42(1):7-15, Jan. 2012.

[24] M. A. Y. Khan and D. Veitch. Speedo: Realistic achievable bandwidth in 802.11 through passive monitoring. In Proc. LCN WNM, pages 892 899,2008

[25] J. Laine, S. Saaristo, and R. Prior. (c)rude. http://rude.sourceforge.net/.

[26] J.-Y. Le Boudec and P. Thiran. Network Calculus A Theory of Deterministic Queuing Systems for the Internet. Springer-Verlag, 2001.

[27] J. Li, M. Claypool, and R. R. Kinicki. Wbest: A bandwidth estimation tool for ieee 802.11 wireless networks. In Proc. IEEE LCN, pages 374 381 , Oct. 2008

[28] J. Liebeherr, M. Fidler, and S. Valaee. A system theoretic approach to bandwidth estimation. IEEE/ACM Trans. Netw., 18(4):1040-1053, Aug. 2010.

[29] X. Liu, K. Ravindran, and D. Loguinov. A queuing-theoretic foundation of available bandwidth estimation: Single-hop analysis. IEEE/ACM Trans. Netw., 15(4):918-931, Aug. 2007.

[30] X. Liu, K. Ravindran, and D. Loguinov. A stochastic foundation of available bandwidth estimation: Multi-hop analysis. IEEE/ACM Trans. Netw., 16(1):130-143, Apr. 2008.

[31] R. Lübben, M. Fidler, and J. Liebeherr. A foundation for stochastic bandwidth estimation of networks with random service. Technical Report arXiv:1008.0050v1 [cs.NI], July 2010.

[32] R. Lübben, M. Fidler, and J. Liebeherr. A foundation for stochastic bandwidth estimation of networks with random service. In Proc. IEEE INFOCOM, pages 1817-1825, Apr. 2011.

[33] S. Machiraju, D. Veitch, F. Baccelli, and J. Bolot. Adding definition to active probing. ACM SIGCOMM CCR, 37(2):19-28, Apr. 2007.

[34] B. Melander, M. Björkman, and P. Gunningberg. A new end-to-end probing and analysis method for estimating bandwidth bottlenecks. In Proc. IEEE GLOBECOM, pages 415-420, Nov. 2000.

[35] B. Melander, M. Björkmann, and P. Gunningberg. First-come-first- served packet dispersion and implications for TCP. In Proc. IEEE GLOBECOM, pages 2170-2174, Nov. 2002.

[36] K.-J. Park, H. Lim, and C.-H. Choi. Stochastic analysis of packetpair probing for network bandwidth estimation. Computer Networks, 50(12):1901-1915, May 2006.

[37] K.-J. Park, H. Lim, J. C. Hou, and C.-H. Choi. Feedback-assisted robust estimation of available bandwidth. Computer Networks, 53(7):896-912, May 2009.

[38] M. Portoles-Comeras, A. Cabellos-Aparicio, J. Mangues-Bafalluy, A. Banchs, and J. Domingo-Pascual. Impact of transient csma/ca access delays on active bandwidth measurements. In Proc. ACM IMC, pages 397-409, Nov. 2009.

[39] V. Ribeiro, R. Riedi, R. Baraniuk, J. Navratil, and L. Cottrell. PathChirp: Efficient available bandwidth estimation for network paths. In Proc. PAM, Apr. 2003.

[40] H. She, Z. Lu, A. Jantsch, and L.-R. Zheng. Estimation of statistical bandwidth through backlog measurement. In Proc. WoNeCa, Mar. 2012.

[41] J. Strauss, D. Katabi, and F. Kaashoek. A measurement study of available bandwidth estimation tools. In Proc. ACM IMC, pages 3944, Oct. 2003.

[42] B. White et al. An integrated experimental environment for distributed systems and networks. In Proc. OSDI, pages 255-270, Dec. 2002.

[43] J. Xie and Y. Jiang. Stochastic service guarantee analysis based on time-domain models. In Proc. IEEE MASCOTS, pages 472-483, Sept. 2009.

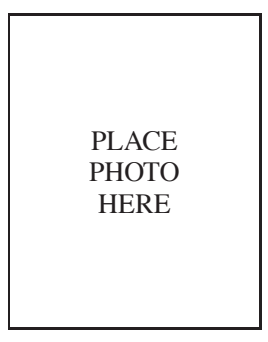

Ralf Lübben (S11) received his master degree in computer science from the Leibniz Universität Hannover, Hannover, Germany, in 2007. Since 2009 he has been a Ph.D. candidate at the information technology institute at Leibniz Universität Hannover, Hannover, Germany.

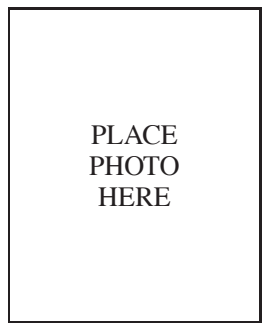

Markus Fidler (M04-SM08) received the doctoral degree in computer engineering from RWTH Aachen University, Germany, in 2004. He was a PostDoctoral Fellow of NTNU Trondheim, Norway, in 2005 and the University of Toronto, ON, Canada, in 2006. During 2007 and 2008, he was an Emmy Noether Research Group Leader at Technische Universität Darmstadt, Germany. Since 2009, he has been a Professor of communications networks at Leibniz Universität Hannover, Germany.

Jörg Liebeherr (S88-M92-SM03-F08) received the Ph.D. degree in computer science from the Georgia Institute of Technology, Atlanta, in 1991. Computer Science at the University of Virginia, Charlottesville, from 1992 to 2005. Since Fall 2005 HERE he has been with the University of Toronto, Toronto, ON, Canada, as Professor of electrical and computer engineering and Nortel Chair of Network Architecture and Services. 